\title{
Vanderbilt-Ingram Cancer Center
}

National Cancer Institute

\section{Source}

National Cancer Institute. Vanderbilt-Ingram Cancer Center. NCI Thesaurus. Code C39534.

The mission of the Vanderbilt-Ingram Cancer Center is to alleviate cancer death and suffering through pioneering research, clinical trials, evidence-based patient-centered care, prevention and education, and community activities. The Center's research programs encompass basic, clinical, and population-based research, interdisciplinary collaboration, and bi-directional translation. It became an $\mathrm{NCl}$-designated cancer center in 1995 and earned its status as a comprehensive cancer center in 2001. 